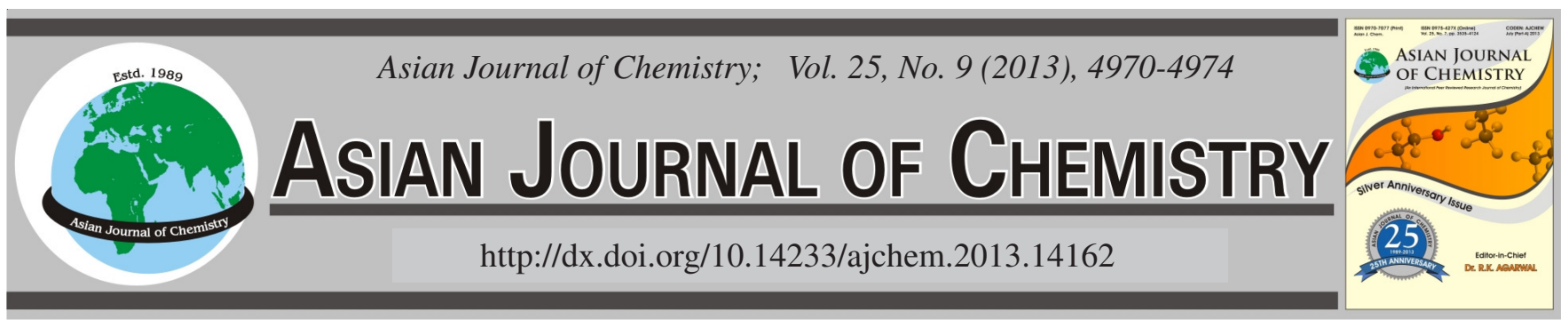

\title{
Synthesis and Anticoccidial Activities of Novel N-(2-Aminophenyl)-2-quinazolinone-acetamide Hydrochloride
}

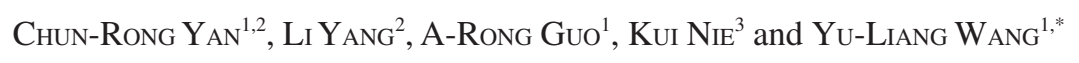

${ }^{1}$ College of Chemistry, Sichuan University, Chengdu 610064, Sichuan Province, P.R. China

${ }^{2}$ College of Chemistry and Chemical Engineering, Yibin University, Yinbin 644000, P.R. China

${ }^{3}$ College of Animal Science and Technology, Southwest University, Chongqing 6400715, P.R. China

*Corresponding author: Tel: +86 15378195811; E-mail: chemwang2008@163.com; allendk@163.com

(Received: 6 June 2012;

Accepted: 13 March 2013)

AJC-13115

Eight novel N-(2-aminophenyl)-2-quinazolinone-acetamide hydrochloride were synthesized and their structures were identified by ${ }^{1} \mathrm{H}$ NMR, MS and IR spectra. Seven of the new compounds were chosen for anticoccidial activity test and the results showed that $\mathrm{N}-(2-$ aminophenyl)-2-(6-methyl-8-bromo quinazolinone)acetamide hydrochloride (3h) exhibited anticoccidial activity against Eimeria tenella in the chicken' diet with a dose of $18 \mathrm{mg} / \mathrm{Kg}$.

Key Words: N-(2-Aminophenyl)-2-quinazolinone-acetamide hydrochloride, Anticoccidial activity, Eimeria tenella.

\section{INTRODUCTION}

Coccidiosis is an intestinal infection caused by protozoan parasites of the genus Eimeria resulting in intestinal lesions, diarrhoea, enteritis and death ${ }^{1,2}$, which occurs all over the world and leads to extensive loss in the poultry industry ${ }^{3}$. Many drugs are effective in the prevention and treatment of coccidiosis, but the coccidia are inevitably to develop resistance to them ${ }^{4,5}$. And the drug resistance has developed almost to all of the anticoccidial drugs introduced so far $^{5}$. Therefore, the search and discovery of new anticoccidial activity drugs to be needed urgently.

Our team designed and synthesized some anticoccidial compounds based on the structures of hydroxyquinolinecarboxylates and febrifugine, respectively. And we found some of them have anticoccidial activities ${ }^{6-13}$.

Febrifugine (Fig. 1, compound 1) have anticoccidial activity which is the effective component of Dichroa febrifuga.
American Cyanamid Company designed and synthesized a anticoccidial drug---halofuginone based on the structure of febrifugine in $1967^{14,15}$. Halofuginone (Fig. 1, compound 2) is a high anticoccidial activity, broad-spectrum and low toxicity anticoccidial drug. However, the synthetic route of halofuginone is long and the raw material is hard to synthesized, which leads to a high cost and limits its application.

In order to find novel and high anticoccidial activities drugs with easy synthesis and lower cost, You et al. ${ }^{11,12}$, Zhan et $a l .{ }^{13}$ designed and synthesized some quinazolinone derivatives, respectively based on the structure of febrifugine. The anticoccidial activities of these compounds were evaluated and the results indicated that these compounds exhibited anticoccidial activities against Eimeria tenella. In this paper, eight novel N-(2-aminophenyl)-2-quinazolinone-acetamide hydrochloride (Fig. 1, compound 3 ) were designed and synthesized according to the structure of febrifugine. The synthesis route is showed in Scheme-I. The structure characteristics of<smiles></smiles><smiles></smiles>

2<smiles></smiles>

$\mathrm{R}^{1}$ or $\mathrm{R}^{2}=\mathrm{H}, \mathrm{F}, \mathrm{Br}, \mathrm{CH}_{3}$

Fig. 1. Structures of compounds 1-3 
the new compounds are as follows: first, the quinazolinone ring of febrifugine is retained; second, the piperidine ringacetone is replaced by $\mathrm{N}$-(2-aminophenyl)-acetamide. The structures of the new compounds are similar with halofuginone. Therefore, the recognitions between molecules and enzymes in the coccidia bodies should also be similar with halofuginone. The new compounds would have anticoccidial activities. Seven of them were chosen for anticoccidial activity test according to the ACI method.

\section{EXPERIMENTAL}

Solvents and reagents were obtained from commercial sources and used without further purification. Melting point was recorded on XRC-1 apparatus and the thermometer was uncorrected. Proton NMR spectra were recorded on a varian Unity Inova-400 spectrometer with DMSO- $d_{6}$ as the solvent and TMS as the internal standard and mass spectra on Agilent 6210 Series Time of Flight LC/MS using the ESI source. IR spectra were recorded with Perkin-Elmer 16PC-FT instrument. Analytical thin-layer chromatography was carried out on precoated plates (silica gel $\mathrm{GF}_{254}$ ) and spots were visualized with ultraviolet light.

General procedure for preparation of $\mathbf{4}^{\mathbf{1 6}}$ : Bromoacetyl chloride $(7.12 \mathrm{~g}, 45.2 \mathrm{mmol})$ was added dropwise to a solution of $o$-nitroaniline $(5.80 \mathrm{~g}, 42.0 \mathrm{mmol})$ in dichloromethane $(60$ $\mathrm{mL})$ at $0{ }^{\circ} \mathrm{C}$. Then, pyridine $(3.32 \mathrm{~g}, 42.0 \mathrm{mmol})$ was slowly added dropwise at $0{ }^{\circ} \mathrm{C}$. The resulting mixture was stirred for $5 \mathrm{~h}$ at room temperature. And the mixture was washed with $3 \mathrm{~mL} \times 20 \mathrm{~mL}$ brine and $3 \mathrm{~mL} \times 20 \mathrm{~mL}$ water and dried over anhydrous $\mathrm{MgSO}_{4}$. After evaporation of solvent, the crude products were obtained and were recrystallized in a mixed solution of ethanol and water to provide intermediates $\mathbf{4}$ in $74.1 \%$ yield.

General procedure for preparation of 5a-h: The compounds $\mathbf{5 a - h}$ were synthesized using the similar reaction conditions in reference ${ }^{11}$.

8-Fluoroquinazolin-4(3H)-one (5a): Brown solid; m.p. $271-273{ }^{\circ} \mathrm{C}$ (lit. ${ }^{17} 272-273{ }^{\circ} \mathrm{C}$ ).
6-Bromo-8-fluoroquinazolin-4(3H)-one $(5 \mathrm{~b})$ : Dark brown solid; m.p. $>300{ }^{\circ} \mathrm{C}$; HR-MS (ESI): Calcd. (\%) for $\mathrm{C}_{8} \mathrm{H}_{9} \mathrm{BrFN}_{2} \mathrm{O}^{+}(\mathrm{M}+\mathrm{H})^{+}: 242.9557,244.9543$ (1:1). The mass spectrum data correspond to lit. ${ }^{18}$.

6-Fluoroquinazolin-4(3H)-one $(5 \mathrm{c})$ : Brown solid; m.p. 248-249 ${ }^{\circ} \mathrm{C}$ (lit. $\left.{ }^{19} 250{ }^{\circ} \mathrm{C}\right)$.

6-Fluoro-8-bromoquinazolin-4(3H)-one (5d): Yellowishbrown solid; m.p. $>300{ }^{\circ} \mathrm{C}$; HR-MS (ESI): Calcd. (\%) for $\mathrm{C}_{8} \mathrm{H}_{9} \mathrm{BrFN}_{2} \mathrm{O}^{+}(\mathrm{M}+\mathrm{H})^{+}: 242.9565,244.9547$ (1:1). The mass spectrum data correspond to lit. ${ }^{20}$.

8-Methylquinazolin-4(3H)-one (5e): Yellow solid; m.p. 247-249 ${ }^{\circ} \mathrm{C}$ (lit. $\left.{ }^{19} 249{ }^{\circ} \mathrm{C}\right)$.

6-Bromo-8-methylquinazolin-4(3H)-one (5f): Brown solid; m.p. > $300{ }^{\circ} \mathrm{C}$; ${ }^{1} \mathrm{H}$ NMR spectrum (400 MHz; DMSO$d_{6}$; TMS $): \delta(\mathrm{ppm})=12.47(\mathrm{~s}, 1 \mathrm{H}), 8.17(\mathrm{~s}, 1 \mathrm{H}), 8.04(\mathrm{~d}, J=$ $2.0 \mathrm{~Hz}, 1 \mathrm{H}), 7.89$ (s, 1H), $2.53(\mathrm{~s}, 3 \mathrm{H})$; IR $\left(\mathrm{KBr}, v_{\max }, \mathrm{cm}^{-1}\right)$ 3364, 3199, 3079, 2884, 2631, 2362, 1945, 1861, 1777, 1683, 1609, 1456, 1403, 1326, 1271, 1220, 1162, 1048, 1002, 947, 888, 837, 797, 699, 595, 508, 480; HR-MS (ESI): Calcd. (\%) for $\mathrm{C}_{9} \mathrm{H}_{8} \mathrm{BrN}_{2} \mathrm{O}^{+}(\mathrm{M}+\mathrm{H})^{+}$: 238.9815; found (\%): 238.9816 , 240.9798(1: 1).

6-Methylquinazolin-4(3H)-one (5g): Ivory-white solid; m.p. $253-255^{\circ} \mathrm{C}$ (lit. ${ }^{19} 256^{\circ} \mathrm{C}$ ).

8-Bromo-6-methylquinazolin-4(3H)-one $(5 \mathrm{~h})^{21}$ : Yellow brown solid; m.p. > $300{ }^{\circ} \mathrm{C}$; ${ }^{1} \mathrm{H}$ NMR spectrum $(400 \mathrm{MHz}$; DMSO- $d_{6}$; TMS $): \delta(\mathrm{ppm})=12.43(\mathrm{~s}, 1 \mathrm{H}), 8.16(\mathrm{~s}, 1 \mathrm{H}), 8.010$ (s, 1H), 7.93 (s, 1H), 2.43 (s, 3H); IR (KBr, $\left.v_{\max }, \mathrm{cm}^{-1}\right) 3359$, 3184, 3063, 2919, 2624, 1944, 1777, 1679, 1606, 1543, 1459, 1397, 1321, 1272, 1250, 1213, 1144, 1086, 1038, 904, 870, 798, 741, 605, 566, 530, 442; HR-MS (ESI): Calcd. (\%) for $\mathrm{C}_{9} \mathrm{H}_{8} \mathrm{BrN}_{2} \mathrm{O}^{+}(\mathrm{M}+\mathrm{H})^{+}:$238.9815; found (\%): 238.9808, 240.9794(1: 1).

General procedure for preparation of 6a-h: A solution of 4 (11.7 mmol) in $15 \mathrm{~mL}$ DMSO was added dropwise to a mixture of 5a-h ${ }^{11}$ (9.33 mmol), KI (0.5 g), NaH (0.28 g, 11.7 $\mathrm{mmol}$ ) and $40 \mathrm{~mL}$ DMSO under stirring at $75^{\circ} \mathrm{C}$. The reaction mixture was stirred at this temperature for $1.5-3.0 \mathrm{~h}$ and then cooled to room temperature. Water $(50 \mathrm{~mL})$ was added to the<smiles>[R]c1cc([12OH])cc2c(=O)[nH]cnc12</smiles>

4<smiles>[R7]c1cc([R])c2ncn(CC(=O)Nc3cccc([N+](=O)[O-])c3[N+](=O)[O-])c(=O)c2c1</smiles>

3a: $R^{1}=H, R^{2}=F ; \quad 3 b: R^{1}=B r, R^{2}=F ; \quad 3 c: R^{1}=F, R^{2}=H ; \quad$ 3d: $R^{1}=F, R^{2}=B r ;$ 3e: $R^{1}=H, R^{2}=C_{3} ; 3 f: R^{1}=B r, R^{2}=C_{3} ; 3 g: R^{1}=C_{3}, R^{2}=H ; 3 h: R^{1}=C_{3}, R^{2}=B r$.

Scheme-I: General synthesis route for compounds $\mathbf{3 a - h}$ 
mixture and the obtained solid was filtrated and recrystallized with ethanol to give $\mathbf{6 a - h}$ in $81-87 \%$ yield. The physical and spectra data of the compounds $\mathbf{6 a - h}$ are as follows.

N-(2-Nitrophenyl)-2-(8-fluoro-quinazolinone)acetamide (6a): Light-yellow solid; yield: $84 \%$; m.p. $220-221{ }^{\circ} \mathrm{C} ;{ }^{1} \mathrm{H}$ NMR spectrum (400 MHz; $\mathrm{CDCl}_{3}-d_{6}$; TMS): $\delta(\mathrm{ppm})=10.82$ $(\mathrm{s}, 1 \mathrm{H}), 8.72(\mathrm{~d}, J=8.4 \mathrm{~Hz}, 1 \mathrm{H}), 8.25(\mathrm{~d}, J=7.6 \mathrm{~Hz}, 1 \mathrm{H})$, 8.14-8.11 (m, 2H), $7.67(\mathrm{t}, J=7.6 \mathrm{~Hz}, 1 \mathrm{H}), 7.57-7.47(\mathrm{~m}$, $2 \mathrm{H}), 7.24(\mathrm{~d}, J=8.0 \mathrm{~Hz}, 1 \mathrm{H}), 4.88(\mathrm{~s}, 2 \mathrm{H})$; IR $\left(\mathrm{KBr}, v_{\max }, \mathrm{cm}^{-1}\right)$ 3439, 3282, 1676, 1609, 1520, 1479, 1366, 1338, 1284, 1254, 1152, 1035, 947, 788, 763; HR-MS (ESI): Calcd. (\%) for $\mathrm{C}_{16} \mathrm{H}_{12} \mathrm{FN}_{4} \mathrm{O}_{4}^{+}(\mathrm{M}+\mathrm{H})^{+}: 343.0837$; found (\%): 343.0830 .

N-(2-Nitrophenyl)-2-(6-bromo-8-fluoro-quinazolinone)acetamide (6b): Light-yellow solid; yield: 86 \%; m.p. 208$209{ }^{\circ} \mathrm{C}$; ${ }^{1} \mathrm{H}$ NMR spectrum (400 MHz; $\mathrm{CDCl}_{3}-d_{6}$; TMS): $\delta$ $(\mathrm{ppm})=10.81(\mathrm{~s}, 1 \mathrm{H}), 8.71(\mathrm{~d}, J=8.4 \mathrm{~Hz}, 1 \mathrm{H}), 8.25(\mathrm{~d}, J=$ $8.0 \mathrm{~Hz}, 2 \mathrm{H}), 8.13$ (s, 1H), 7.67 (t, $J=7.2 \mathrm{~Hz}, 2 \mathrm{H}), 7.28-7.24$ $(\mathrm{m}, 1 \mathrm{H}), 4.88$ (s, 2H); IR (KBr, $\left.v_{\max }, \mathrm{cm}^{-1}\right) 3433,3298,1693$, 1608, 1507, 1469, 1363, 1331, 1276, 1250, 1177, 1046, 966, 867, 797, 745, 714; HR-MS (ESI): Calcd. (\%) for $\mathrm{C}_{16} \mathrm{H}_{11} \mathrm{BrFN}_{4} \mathrm{O}_{4}{ }^{+}(\mathrm{M}+\mathrm{H})^{+}:$420.9942; found (\%): 420.9950, 422.9928(1:1).

N-(2-Nitrophenyl)-2-(6-fluoro-quinazolinone)acetamide (6c): Light-yellow solid; yield: $83 \%$; m.p. 196-198 ${ }^{\circ} \mathrm{C}$; ${ }^{1} \mathrm{H}$ NMR spectrum (400 MHz; $\mathrm{CDCl}_{3}-d_{6}$; TMS): $\delta(\mathrm{ppm})=$ $10.81(\mathrm{~s}, 1 \mathrm{H}), 8.72(\mathrm{~d}, J=8.4 \mathrm{~Hz}, 1 \mathrm{H}), 8.24(\mathrm{~d}, J=8.4 \mathrm{~Hz}$, $1 \mathrm{H}), 8.10(\mathrm{~s}, 1 \mathrm{H}), 7.97\left(\mathrm{dd}, J_{1}=2.8 \mathrm{~Hz}, J 2=8.4 \mathrm{~Hz}, 1 \mathrm{H}\right)$, $7.80\left(\mathrm{dd}, J_{1}=4.4 \mathrm{~Hz}, J_{2}=4.8 \mathrm{~Hz}, 1 \mathrm{H}\right), 7.67(\mathrm{t}, J=8.0 \mathrm{~Hz}$, $1 \mathrm{H}), 7.53\left(\mathrm{td}, J_{1}=2.8 \mathrm{~Hz}, J_{2}=8.4 \mathrm{~Hz}, 1 \mathrm{H}\right), 7.24(\mathrm{~d}, J=7.6$ $\mathrm{Hz}, 1 \mathrm{H}), 4.88$ (s, 2H); IR (KBr, $\left.v_{\max }, \mathrm{cm}^{-1}\right) 3432,3317,1673$, 1613, 1587, 1544, 1510, 1483, 1404, 1349, 1280, 1158, 964, 847, 787, 743, 708, 621; HR-MS (ESI): Calcd. (\%) for $\mathrm{C}_{16} \mathrm{H}_{12} \mathrm{FN}_{4} \mathrm{O}_{4}^{+}(\mathrm{M}+\mathrm{H})^{+}$: 343.0837 ; found (\%): 343.0841.

N-(2-Nitrophenyl)-2-(6-fluoro-8-bromo-quinazolinone)acetamide (6d): Yellow solid; yield: $87 \%$; m.p. $251-252{ }^{\circ} \mathrm{C}$; ${ }^{1} \mathrm{H}$ NMR spectrum (400 MHz; $\left.\mathrm{CDCl}_{3}-d_{6} ; \mathrm{TMS}\right): \delta(\mathrm{ppm})=$ $10.81(\mathrm{~s}, 1 \mathrm{H}), 8.71(\mathrm{~d}, J=8.4 \mathrm{~Hz}, 1 \mathrm{H}), 8.25\left(\mathrm{dd}, J_{1}=1.6 \mathrm{~Hz}\right.$, $\left.J_{2}=8.4 \mathrm{~Hz}, 1 \mathrm{H}\right), 8.17(\mathrm{~s}, 1 \mathrm{H}), 7.97\left(\mathrm{dd}, J_{1}=2.8 \mathrm{~Hz}, J_{2}=8.0\right.$ $\mathrm{Hz}, 1 \mathrm{H}), 7.88\left(\mathrm{dd}, J_{1}=2.8 \mathrm{~Hz}, J_{2}=8.4 \mathrm{~Hz}, 1 \mathrm{H}\right), 7.67(\mathrm{t}, J=$ $8.4 \mathrm{~Hz}, 1 \mathrm{H}), 7.27-7.23(\mathrm{~m}, 1 \mathrm{H}), 4.88$ (s, 2H); IR (KBr, $\mathrm{v}_{\max }$, $\mathrm{cm}^{-1}$ ) 3434, 3298, 3074, 1687, 1611, 1552, 1513, 1465, 1386, 1440, 1282, 1164, 961, 878, 795, 739, 630; HR-MS (ESI): Calcd. (\%) for $\mathrm{C}_{16} \mathrm{H}_{11} \mathrm{BrFN}_{4} \mathrm{O}_{4}^{+}(\mathrm{M}+\mathrm{H})^{+}$: 420.9942; found (\%): 420.9936, 422.9920(1:1).

N-(2-Nitrophenyl)-2-(8-methyl-quinazolinone)-acetamide (6e): Light-yellow solid; yield: $80 \%$; m.p. $210-212{ }^{\circ} \mathrm{C}$; ${ }^{1} \mathrm{H}$ NMR spectrum (400 MHz; $\left.\mathrm{CDCl}_{3}-d_{6} ; \mathrm{TMS}\right): \delta(\mathrm{ppm})=$ $10.81(\mathrm{~s}, 1 \mathrm{H}), 8.72$ (d, $J=8.4 \mathrm{~Hz}, 1 \mathrm{H}), 8.24-8.18(\mathrm{~m}, 2 \mathrm{H})$, $8.12(\mathrm{~s}, 1 \mathrm{H}), 7.67-7.63(\mathrm{~m}, 2 \mathrm{H}), 7.43(\mathrm{t}, J=8.0 \mathrm{~Hz}, 1 \mathrm{H}), 7.21$ (t, $J=8.0 \mathrm{~Hz}, 1 \mathrm{H}), 4.87(\mathrm{~s}, 2 \mathrm{H}), 2.65(\mathrm{~s}, 3 \mathrm{H})$; IR (KBr, $v_{\max }$, $\left.\mathrm{cm}^{-1}\right)$ 3433, 3271, 1688, 1609, 1550, 1514, 1371, 1337, 1283, 1154, 962, 775, 734; HR-MS (ESI): Calcd. (\%) for $\mathrm{C}_{17} \mathrm{H}_{15} \mathrm{~N}_{4} \mathrm{O}_{4}{ }^{+}$ $(\mathrm{M}+\mathrm{H})^{+}: 339.1088$; found $(\%): 339.1089$.

N-(2-Nitrophenyl)-2-(6-bromo-8-methyl-quinazolinone)acetamide (6f): Yellow solid; yield: $82 \%$; m.p. 236-238 ${ }^{\circ} \mathrm{C}$; ${ }^{1} \mathrm{H}$ NMR spectrum (400 MHz; $\mathrm{CDCl}_{3}-d_{6}$; TMS): $\delta(\mathrm{ppm})=$ $10.80(\mathrm{~s}, 1 \mathrm{H}), 8.71(\mathrm{~d}, J=8.4 \mathrm{~Hz}, 1 \mathrm{H}), 8.31$ (d, $J=2.0 \mathrm{~Hz}$, $1 \mathrm{H}), 8.24\left(\mathrm{dd}, J_{1}=1.2 \mathrm{~Hz}, J_{2}=8.4 \mathrm{~Hz}, 1 \mathrm{H}\right), 8.16(\mathrm{~s}, 1 \mathrm{H}), 7.76$ $\left(\mathrm{d}, J_{1}=1.6 \mathrm{~Hz}, 1 \mathrm{H}\right), 7.66(\mathrm{t}, J=7.6 \mathrm{~Hz}, 1 \mathrm{H}), 7.24-7.22(\mathrm{~m}$,
$1 \mathrm{H}), 4.88(\mathrm{~s}, 2 \mathrm{H}), 2.63(\mathrm{~s}, 3 \mathrm{H})$; IR $\left(\mathrm{KBr}, \mathrm{v}_{\max }, \mathrm{cm}^{-1}\right) 3432$, 3309, 1701, 1610, 1555, 1513, 1459, 1337, 1283, 1162, 962, 877, 796, 741, 633; HR-MS (ESI): Calcd. (\%) for $\mathrm{C}_{17} \mathrm{H}_{14} \mathrm{BrN}_{4} \mathrm{O}_{4}{ }^{+}(\mathrm{M}+\mathrm{H})^{+}$: 417.0193; found $(\%)$ : 417.0180, 419.0165(1:1).

N-(2-Nitrophenyl)-2-(6-methyl-quinazolinone)acetamide (6g): Ivory-white solid; yield: 78 \%; m.p. 232-234 ${ }^{\circ} \mathrm{C}$; ${ }^{1} \mathrm{H}$ NMR spectrum (400 MHz; $\mathrm{CDCl}_{3}-d_{6}$; TMS): $\delta(\mathrm{ppm})=$ $10.81(\mathrm{~s}, 1 \mathrm{H}), 8.72(\mathrm{~d}, J=8.4 \mathrm{~Hz}, 1 \mathrm{H}), 8.23(\mathrm{~d}, J=8.4 \mathrm{~Hz}$, $1 \mathrm{H}), 8.13(\mathrm{~s}, 1 \mathrm{H}), 8.09$ (s, 1H), $7.68(\mathrm{~d}, J=8.0 \mathrm{~Hz}, 1 \mathrm{H}), 7.64$ $(\mathrm{t}, J=7.6 \mathrm{~Hz}, 2 \mathrm{H}), 7.23(\mathrm{t}, J=8.0 \mathrm{~Hz}, 1 \mathrm{H}), 4.87(\mathrm{~s}, 2 \mathrm{H}), 2.51$ (s, 3H); IR (KBr, $\left.v_{\max }, \mathrm{cm}^{-1}\right) 3429,3238,1689,1609,1522$, 1382, 1340, 1283, 1166, 962, 838, 738; HR-MS (ESI): Calcd. (\%) for $\mathrm{C}_{17} \mathrm{H}_{15} \mathrm{~N}_{4} \mathrm{O}_{4}{ }^{+}(\mathrm{M}+\mathrm{H})^{+}: 339.1088$; found (\%): 339.1083.

N-(2-Nitrophenyl)-2-(6-methyl-8-bromo-quinazolinone)acetamide (6h): Light-yellow solid; yield: $81 \%$; m.p. 261$263{ }^{\circ} \mathrm{C}$; ${ }^{1} \mathrm{H}$ NMR spectrum (400 MHz; $\mathrm{CDCl}_{3}-d_{6}$; TMS): $\delta$ $(\mathrm{ppm})=10.81(\mathrm{~s}, 1 \mathrm{H}), 8.72(\mathrm{~d}, J=8.4 \mathrm{~Hz}, 1 \mathrm{H}), 8.25(\mathrm{~s}, 1 \mathrm{H})$, $8.23(\mathrm{~s}, 1 \mathrm{H}), 8.10(\mathrm{~s}, 1 \mathrm{H}), 7.93(\mathrm{~s}, 1 \mathrm{H}), 7.66(\mathrm{t}, J=8.0 \mathrm{~Hz}$, $1 \mathrm{H}), 7.23$ (d, $J=8.0 \mathrm{~Hz}, 1 \mathrm{H}), 4.89$ (s, 2H), 2.49 (s, 3H); IR (KBr, $\left.v_{\max }, \mathrm{cm}-1\right) 3431,3287,2920,1686,1610,1511,1466$, 1367, 1339, 1281, 1169, 958, 855, 795, 738; HR-MS (ESI): Calcd. (\%) for $\mathrm{C}_{17} \mathrm{H}_{14} \mathrm{BrN}_{4} \mathrm{O}_{4}{ }^{+}(\mathrm{M}+\mathrm{H})^{+}$: 417.0193; found (\%): 417.0182, 419.0167(1:1).

General procedure for preparation of $\mathbf{3 a - h}$ : A mixture of 6a-h (1.66 mmol), Fe (0.28 g, $5.00 \mathrm{mmol})), \mathrm{NH}_{4} \mathrm{Cl}(0.80 \mathrm{~g}$, $15.00 \mathrm{mmol})$, tetrabutylammonium bromide $(0.30 \mathrm{~g})$, ethyl alcohol $(25 \mathrm{~mL})$ and water $(5 \mathrm{~mL})$ was stirred and refluxed at $70{ }^{\circ} \mathrm{C}$ for $0.5-2.0 \mathrm{~h}$. The mixture was filtered after cooled to room temperature then red-brown solid was obtained. This solid was dissolved in DMSO (10-20 mL) and then filtered. Yellow filtrate was retained. Water $(100 \mathrm{~mL})$ was added to the filtrate and a lot of solid appeared immediately. The resulting precipitate was filtered and acidified with $\mathrm{HCl}$ to give $\mathbf{3 a}-\mathbf{h}$ in 69-81\% yield. The physical and spectra data of the compounds $\mathbf{3 a - h}$ are as follows.

N-(2-Aminophenyl)-2-(8-fluoro-quinazolinone)acetamide hydrochloride (3a): Light-yellow solid; yield: $72 \%$; m.p. 207-210 ${ }^{\circ} \mathrm{C}$; ${ }^{1} \mathrm{H}$ NMR spectrum $\left(400 \mathrm{MHz}\right.$; DMSO- $d_{6}$; TMS): $\delta(\mathrm{ppm})=10.59(\mathrm{~s}, 1 \mathrm{H}), 8.47(\mathrm{~s}, 1 \mathrm{H}), 7.98(\mathrm{~d}, J=7.6$ $\mathrm{Hz}, 1 \mathrm{H}), 7.78-7.73(\mathrm{~m}, 1 \mathrm{H}), 7.57\left(\mathrm{dt}, J_{1}=4.8 \mathrm{~Hz}, J_{2}=8.0\right.$ $\mathrm{Hz}, 1 \mathrm{H}), 7.37$ (d, $J=7.6 \mathrm{~Hz}, 1 \mathrm{H}), 7.30-7.15$ (m, 3H), 4.98 (s, 2H), 6.00-3.60 (s, 3H); IR (KBr, $\left.v_{\max }, \mathrm{cm}^{-1}\right) 3421,3232,2986$, 2823, 2591, 2021, 1910, 1800, 1720, 1654, 1618, 1544, 1498, 1423, 1391, 1366, 1344, ,1296, 1251, 1218, 1185, 1175, 1155,1109, 1024, 963, 934, 908, 878, 839, 795, 745, 707, 654, 610, 574, 549, 501, 466; HR-MS (ESI): Calcd. (\%) for $\mathrm{C}_{16} \mathrm{H}_{14} \mathrm{FN}_{4} \mathrm{O}_{2}{ }^{+}(\mathrm{M}-\mathrm{Cl})^{+}:$313.1095; found (\%): 313.1091 .

$\mathrm{N}$-(2-Aminophenyl)-2-(6-bromo-8-fluoro-quinazolinone)acetamide hydrochloride (3b): Ivory-white solid; yield: 74 \%; m.p. 221-224 ${ }^{\circ} \mathrm{C}$; ${ }^{1} \mathrm{H}$ NMR spectrum (400 MHz; DMSO$d_{6}$; TMS $): \delta(\mathrm{ppm})=10.38(\mathrm{~s}, 1 \mathrm{H}), 8.52(\mathrm{~s}, 1 \mathrm{H}), 8.12-8.09(\mathrm{~m}$, $2 \mathrm{H}), 7.33(\mathrm{~d}, J=7.6 \mathrm{~Hz}, 1 \mathrm{H}), 7.20-7.00(\mathrm{~m}, 3 \mathrm{H}), 4.97(\mathrm{~s}, 2 \mathrm{H})$, 5.70-3.30 (s, 3H); IR (KBr, $\left.v_{\max }, \mathrm{cm}^{-1}\right) 3430,3266,3117,3078$, 2828, 2542, 1949, 1898, 1687, 1611, 1560, 1530, 1478, 1393, 1360, 1332, 1286, 1254, 1212, 1169, 1111, 1054, 966, 911, 867, 808, 788, 760, 680, 613, 570, 548, 493, 451; HR-MS (ESI): Calcd. (\%) for $\mathrm{C}_{16} \mathrm{H}_{13} \mathrm{BrFN}_{4} \mathrm{O}_{2}^{+}(\mathrm{M}-\mathrm{Cl})^{+}: 391.0200$; found (\%): 391.0193, $393.0177(1: 1)$. 
N-(2-Aminophenyl)-2-(6-fluoro-quinazolinone)acetamide hydrochloride (3c): Yellow solid; yield: $69 \%$; m.p. $217-$ $220{ }^{\circ} \mathrm{C} ;{ }^{1} \mathrm{H}$ NMR spectrum (400 MHz; DMSO- $d_{6}$; TMS): $\delta$ $(\mathrm{ppm})=10.71(\mathrm{~s}, 1 \mathrm{H}), 8.44(\mathrm{~s}, 1 \mathrm{H}), 7.86-7.75(\mathrm{~m}, 3 \mathrm{H}), 7.46-$ $7.42(\mathrm{~m}, 1 \mathrm{H}), 7.34-7.26(\mathrm{~m}, 3 \mathrm{H}), 5.00(\mathrm{~s}, 2 \mathrm{H}), 6.20-4.40(\mathrm{~s}$, $3 \mathrm{H})$; IR (KBr, $\left.v_{\max }, \mathrm{cm}-1\right)$ 3420, 3206, 2824, 2589, 1716, 1666, 1620, 1586, 1560, 1532, 1491, 1457, 1391, 1361, 1294, 1272, $1195,1151,1121,1056,1007,966,905,840,769,744,699$, 643, 569, 544, 522, 493, 453, 424, 403; HR-MS (ESI): Calcd. (\%) for $\mathrm{C}_{16} \mathrm{H}_{14} \mathrm{FN}_{4} \mathrm{O}_{2}{ }^{+}(\mathrm{M}-\mathrm{Cl})^{+}:$313.1095; found (\%): 313.1092 .

N-(2-Aminophenyl)-2-(6-fluoro-8-bromo-quinazolinone)acetamide hydrochloride (3d): Light-yellow solid; yield: 74 $\%$; m.p. 226-230 ${ }^{\circ} \mathrm{C}$; ${ }^{1} \mathrm{H}$ NMR spectrum (400 MHz; DMSO$d_{6}$; TMS $): \delta(\mathrm{ppm})=9.75(\mathrm{~s}, 1 \mathrm{H}), 8.49(\mathrm{~s}, 1 \mathrm{H}), 8.26\left(\mathrm{dd}, J_{1}=\right.$ $\left.2.8 \mathrm{~Hz}, J_{2}=8.4 \mathrm{~Hz}, 1 \mathrm{H}\right), 7.90\left(\mathrm{dd}, J_{1}=2.8 \mathrm{~Hz}, J_{2}=8.4 \mathrm{~Hz}\right.$, $1 \mathrm{H}), 7.15(\mathrm{~d}, J=7.6 \mathrm{~Hz}, 1 \mathrm{H}), 6.98(\mathrm{t}, J=7.2 \mathrm{~Hz}, 1 \mathrm{H}), 6.79(\mathrm{~d}$, $J=8.0 \mathrm{~Hz}, 1 \mathrm{H}), 6.63(\mathrm{t}, J=7.2 \mathrm{~Hz}, 1 \mathrm{H}), 4.90(\mathrm{~s}, 2 \mathrm{H}), 4.20-$ 3.10 (s, 3H); IR (KBr, $\left.v_{\max }, \mathrm{cm}^{-1}\right) 3428,3249,1690,1656$, 1612, 1542, 1499, 1462, 1399, 1328, 1111, 963, 878, 799, 750, 579; HR-MS (ESI): Calcd. (\%) for $\mathrm{C}_{16} \mathrm{H}_{13} \mathrm{BrFN}_{4} \mathrm{O}_{2}{ }^{+}(\mathrm{M}-$ $\mathrm{Cl})^{+}$: 391.0200; found (5): 391.0195, 393.0179 (1:1).

N-(2-Aminophenyl)-2-(8-methyl-quinazolinone)acetamide hydrochloride (3e): Yellow solid; yield: $72 \%$; m.p. $236-240{ }^{\circ} \mathrm{C} ;{ }^{1} \mathrm{H}$ NMR spectrum (400 MHz; DMSO- $d_{6}$; TMS): $\delta(\mathrm{ppm})=10.82(\mathrm{~s}, 1 \mathrm{H}), 8.47(\mathrm{~s}, 1 \mathrm{H}), 8.01(\mathrm{~d}, J=7.2 \mathrm{~Hz}, 1 \mathrm{H})$, $7.73(\mathrm{~d}, J=7.2 \mathrm{~Hz}, 1 \mathrm{H}), 7.47(\mathrm{~d}, J=7.6 \mathrm{~Hz}, 1 \mathrm{H}), 7.46(\mathrm{t}, J=$ $7.6 \mathrm{~Hz}, 1 \mathrm{H}), 7.42-7.28(\mathrm{~m}, 3 \mathrm{H}), 9.00-6.00(\mathrm{~s}, 3 \mathrm{H}), 5.00(\mathrm{~s}$, 2H), 2.57 (s, 3H); IR (KBr, $\left.v_{\max }, \mathrm{cm}^{-1}\right) 3421,2961,2579,1712$, 1658, 1621, 1587, 1544, 1493, 1459, 1384, 1358, 1334, 1293, 1225, 1194, 1156, 1130, 1064, 1034, 990, 915, 863, 761, 733, 802, 670, 599, 540, 488, 459; HR-MS (ESI): Calcd. (\%) for $\mathrm{C}_{17} \mathrm{H}_{17} \mathrm{~N}_{4} \mathrm{O}_{2}^{+}(\mathrm{M}-\mathrm{Cl})^{+}$: 309.1346; found (\%): 309.1343.

N-(2-Aminophenyl)-2-(6-bromo-8-methyl-quinazolinone)acetamide hydrochloride (3f): Light-yellow solid; yield: 70 $\%$; m.p. $210-213{ }^{\circ} \mathrm{C}$; ${ }^{1} \mathrm{H}$ NMR spectrum (400 MHz; DMSO$d_{6}$; TMS $): \delta(\mathrm{ppm})=10.70(\mathrm{~s}, 1 \mathrm{H}), 8.50(\mathrm{~s}, 1 \mathrm{H}), 8.08(\mathrm{~d}, J=$ $2.0 \mathrm{~Hz}, 1 \mathrm{H}), 7.94(\mathrm{~d}, J=1.2 \mathrm{~Hz}, 1 \mathrm{H}), 7.43-7.20(\mathrm{~m}, 4 \mathrm{H})$, 7.20-6.00 (s, 3H), 4.99 (s, 2H), 2.56 (s, 3H); IR (KBr, v $\max$, $\left.\mathrm{cm}^{-1}\right) 3424,2801,2580,1716,1658,1617,1577,1540,1493$, $1459,1385,1358,1331,1289,1221,1195,1159,1129,1040$, 1001, 964, 872, 794, 764, 702, 665, 601, 544, 488, 456; HRMS (ESI): Calcd. (\%) for $\mathrm{C}_{17} \mathrm{H}_{16} \mathrm{BrN}_{4} \mathrm{O}_{2}{ }^{+}(\mathrm{M}-\mathrm{Cl})^{+}: 387.0451$; found (\%): 387.0445, 389.0429 (1: 1)
N-(2-Aminophenyl)-2-(6-methyl-quinazolinone)acetamide hydrochloride (3g): Ivory-white solid; yield: 81 $\%$; m.p. 229-232 ${ }^{\circ} \mathrm{C}$; ${ }^{1} \mathrm{H}$ NMR spectrum (400 MHz; DMSO$d_{6}$; TMS $): \delta(\mathrm{ppm})=10.63(\mathrm{~s}, 1 \mathrm{H}), 8.41(\mathrm{~s}, 1 \mathrm{H}), 7.97(\mathrm{~s}, 1 \mathrm{H})$, $7.70\left(\mathrm{dd}, J_{1}=1.6 \mathrm{~Hz}, J_{2}=8.4 \mathrm{~Hz}, 1 \mathrm{H}\right), 7.63(\mathrm{~d}, J=8.8 \mathrm{~Hz}$, 1H), 7.42-7.40 (m, 1H), 7.30-7.24 (m, 3H), 6.00-4.00 (s, 3H), 4.97 (s, 2H), 2.47 (s, 3H); IR (KBr, $\left.v_{\max }, \mathrm{cm}-1\right) 3421,3267$, 2979, 2960, 2481, 2039, 1922, 1798, 1711, 1671, 1613, 1579, 1535, 1493, 1454, 1379, 1309, 1266, 1224, 1198, 1176, 1137 , 1027, 981, 901, 868, 825, 783, 757, 696, 663, 619, 538, 496, 454, 423; HR-MS (ESI): Calcd. (\%) for $\mathrm{C}_{17} \mathrm{H}_{17} \mathrm{~N}_{4} \mathrm{O}_{2}{ }^{+}(\mathrm{M}-\mathrm{Cl})^{+}$: 309.1346; found (\%): 309.1346.

N-(2-Aminophenyl)-2-(6-methyl-8-bromo-quinazolinone)acetamide hydrochloride (3h): Yellow solid; yield: $75 \%$; m.p. $193-195{ }^{\circ} \mathrm{C}$; ${ }^{1} \mathrm{H}$ NMR spectrum (400 MHz; DMSO- $d_{6}$; TMS): $\delta(\mathrm{ppm})=9.92(\mathrm{~s}, 1 \mathrm{H}), 8.43(\mathrm{~s}, 1 \mathrm{H}), 8.06(\mathrm{~d}, J=1.6$ $\mathrm{Hz}, 1 \mathrm{H}), 7.97$ (s, 1H), 7.20 (d, J=7.2 Hz, 1H), 7.03 (t, $J=7.6$ $\mathrm{Hz}, 1 \mathrm{H}), 6.89$ (d, $J=7.6 \mathrm{~Hz}, 1 \mathrm{H}), 6.80-7.70(\mathrm{~m}, 1 \mathrm{H}), 4.90$ (s, $2 \mathrm{H}), 4.20-3.20(\mathrm{~s}, 3 \mathrm{H}), 2.46(\mathrm{~s}, 3 \mathrm{H})$; IR $\left(\mathrm{KBr}, \mathrm{v}_{\max }, \mathrm{cm}^{-1}\right) 3433$, 3249, 2922, 2535, 1669, 1612, 1528, 1465, 1399, 1127, 754, 616; HR-MS (ESI): Calcd. (\%) for $\mathrm{C}_{17} \mathrm{H}_{16} \mathrm{BrN}_{4} \mathrm{O}_{2}{ }^{+}(\mathrm{M}-\mathrm{Cl})^{+}$: 387.0451; found (\%): 387.0447, 389.0431 (1: 1).

Biological assay: The anticoccidial activities of the compounds 3a-d and $\mathbf{3 f - h}$ were evaluated according to the anticoccidial index method ${ }^{22-24}$, using decoquinate and diclazuril as reference drug. Briefly, the chickens used to test the anticoccidial activity of compounds were brought and fed to 12-day-old by the feed stuff without using any anticoccidial drugs.

Groups of the chickens were randomly housed, with 15 in each cage and 11 cages were randomly assigned by tier. Group 1-9 of 13-day-old chickens were fed the basal starter diet with the compounds 3a-d $(18 \mathrm{mg} / \mathrm{Kg})$ or $\mathbf{3 f}-\mathbf{h}(18 \mathrm{mg} /$ $\mathrm{Kg}$ ) or decoquinate $(27 \mathrm{mg} / \mathrm{Kg})$, or diclazuril ( $1 \mathrm{mg} / \mathrm{Kg})$ until the end of the test. Group1-10 of 14-day-old chickens were infected artificially with the Eimeria tenella spores of the oocysts 100,000 . Held on observation for 7 days after infection, recorded the weight gain, mortality, lesion scores and oocysts scores of the chicken and calculated the anticoccidial index (ACI). Results of test are given in Table-1.

\section{RESULTS AND DISCUSSION}

As shown in Scheme-I, intermediates 6a-h were synthesized by the reaction of intermediate $\mathbf{4}$ and $\mathbf{5 a}-\mathbf{h}$ with the $\mathrm{NaH}$.

TABLE-1

DATA FOR ANTICOCCIDIAL ACTIVITIES OF COMPOUNDS 3a-d, 3f-h, DECOQUINATE AND DICLAZURIL AGAINST Eimeria tenella

\begin{tabular}{|c|c|c|c|c|c|c|}
\hline Test groups & Test compounds $(\mathrm{mg} / \mathrm{Kg})$ & Rate of relative body weight gain & Survival rate $(\%)$ & Lesion scores & Oocyst scores & $\mathrm{ACI}^{\mathrm{a}}$ \\
\hline 1 & $\mathbf{3 a}(18)$ & 46.8 & 100 & 30 & 40 & 76.8 \\
\hline 2 & $\mathbf{3 b}(18)$ & 76.6 & 100 & 28 & 40 & 108.6 \\
\hline 3 & $3 c(18)$ & 34.1 & 100 & 20 & 20 & 94.1 \\
\hline 4 & 3d (18) & 63.9 & 93.3 & 25 & 40 & 92.2 \\
\hline 5 & 3f (18) & 59.6 & 100 & 28 & 40 & 91.6 \\
\hline 6 & $3 \mathbf{g}(18)$ & 46.8 & 100 & 29 & 40 & 77.8 \\
\hline 7 & 3h $(18)$ & 68.1 & 100 & 26 & 20 & 122.1 \\
\hline 8 & Decoquinate (27) & 71.7 & 100 & 13 & 0 & 158.7 \\
\hline 9 & Diclazuril (1) & 53.5 & 93.3 & 20 & 20 & 106.8 \\
\hline 10 & $\mathrm{ING}^{\mathrm{b}}$ & 63.0 & 86.7 & 28 & 40 & 81.7 \\
\hline 11 & $\mathrm{NNG}^{\mathrm{c}}$ & 100 & 100 & 0 & 0 & 200 \\
\hline
\end{tabular}

${ }^{a}$ Anticoccidial activity index. ${ }^{b}$ Infected non-medicated group. ${ }^{~}$ Non-infected non-medicated group. 
To ensure the intermediates $\mathbf{5} \mathbf{a}-\mathbf{h}$ were reacted completely, the intermediate $\mathbf{4}$ must be in excess. And the overdose of intermediate $\mathbf{4}$ will to be removed by the washing with ethanol or ethyl acetate. Using of this method, we can obtain the intermediates 6a-h with good purity and high yield.

Biological activity: The data for anticoccidial activities of the compounds 3a-d and $\mathbf{3 f}-\mathbf{h}$ were shown in Table-1. ACI are calculated on rate of relative body weight gain, survival rate and the lesion, oocyst scores data. In the positive control group, the coccidiosis in chickens was obviously with ACI 81.7. And in the negative control group, no coccidiosis in chickens was occurred. So the control was set up. The result revealed that $\mathrm{N}$-(2-aminophenyl)-2-(6-methyl-8-bromo quinazolinone)acetamide hydrochloride $(\mathbf{3 h})$ has anticoccidial activity against Eimeria tenella with ACI 122.1. And the compound $\mathbf{3 h}$ exhibited effective anticoccidial activity compare with the positive control group.

\section{Conclusion}

Eight novel N-(2-aminophenyl)-2-quinazolinone-acetamide hydrochloride (1:1) were designed and synthesized. And seven of these compounds were chosen for anticoccidial activity test according to the ACI. The results indicated that $\mathrm{N}$-(2-aminophenyl)-2-(6-methyl-8-bromo quinazolinone)acetamide hydrochloride (3h) has anticoccidial activity against Eimeria tenella with ACI 122.1 and exhibited effective anticoccidial activity compared with the positive control group. Further structural optimization and anticoccidial activities about the quinazolinone derivatives are still in progress.

\section{ACKNOWLEDGEMENTS}

The authors appreciated the financial support from the National Science Foundation of China (No. 21072135) and ${ }^{1} \mathrm{H}$ NMR analysis by Sichuan University Analytical \& Testing Center.

\section{REFERENCES}

1. C.F. Duffy, G.F. Mathis and R.F. Power, Vet. Parasitol., 130, 185 (2005).

2. C.H. Yun, H.S. Lillehoj and E.P. Lillehoj, Dev. Comp. Immunol., 24, 303 (2000).

3. V. Cozma and A. Titilincu, CAB Rev., 2, 61 (2007).

4. G. Greif and B. Stephan, Parasitol. Res., 82, 706 (1996).

5. H.D. Chapman, Avian Pathol., 26, 221(1997).

6. C.R. Yan, J. Xu, Y.B. Weng, J. Li, Y.L. Wang and H. Chen, Chem. Biol. Drug Des., 72, 314 (2008).

7. Y.Y. Wang, C.R. Yan, Y.B. W, H. Jin and H. Chen, Pestic. Biochem. Phys., 93, 85 (2009).

8. Y. Zou, C.R. Yan, Y.B. W, J. Li, K.Q. Wu, Y.L. Wang and Y.Z. Wang, J. Chem. Res., 4, 252 (2009).

9. C.R. Yan, X.Y. Zeng, Y.P. Yao, K. Nie, Y.L. Wang and H. Chen, J. Chem. Res., 2, 71 (2010).

10. Z. Wang, L.J. Zhou, Y.L. Wang, Y.B. Weng, J. He and K. Nie, J. Chem. Res., 7, 378 (2011).

11. J.H. You, C.W. Ye, Y.B. Weng, X.H. Mo and Y.L. Wang, ARKIVOC, 1 (2008).

12. C.W. Ye, J.H. You, X.F. Li, R. You, Y.B. Weng, J. Li and Y.L. Wang, Pestic. Biochem. Phys., 97, 194 (2010).

13. Y.Y. Zhan, G.M. Chen, Y.B. Weng, R.L. Li, Y.L. Wang and Y.Z. Wang, Lett. Drug. Des. Discov., 7, 471 (2010).

14. R. Bernard and N.Y. Baker, US Patent 2775597 (1956).

15. W.P. Emanuel, US Patent 3320124 (1967).

16. H. Xie, D. Ng, S.N. Savinov, B. Dey, P.D. Kwong, R. Wyatt, A.B. Smith III and W.A. Hendrickson, J. Med. Chem., 50, 4898 (2007).

17. G.A. Roth and J.J. Tai, J. Heterocycl. Chem., 33, 2051 (1996).

18. L. Baerfacker, R. Kast, N. Griebenow, H. Meier, P. Kolkhof, B. AlbrechtKuepper, A. Nitsche, J.P. Stasch, D. Schneider and N. Teusch, WO Patent 2010020366 (2010).

19. L. Oerfi, F. Waczek, J. Pato, I. Varga, B. Hegymegi-Barakonyi, R.A. Houghten and G. Keri, Curr. Med. Chem., 11, 2549 (2004).

20. S. Inoue, Y. Tsuboi, Y. Ikeda, T. Nakajima, N. Fujimoto, S. Iwasaki and Y. Fukano, WO Patent 2011148922 (2011).

21. W.D. Schmitz, J.J. Bronson, A.P. Degnan and I.E. Whitney, US Patent 2009/0018,163 (2008).

22. T. Tomio, T. Yoshikazu, H. Masa, N. Hiroshi and S. Kiyoshi, US Patent 5215981 (1990).

23. G.Q. Li, S. Kanu, F.Y. Xiang, S.M. Xiao, L. Zhang, H.W. Chen and H.J. Ye, Vet. Parasitol., 119, 261 (2004).

24. S.D. Folz, B.L. Lee, L.H. Nowakowsk, D.L. Rector and B.M. Folz, J. Parasitol., 75, 696 (1989) 